Utah State University

DigitalCommons@USU

Mechanical and Aerospace Engineering Student Mechanical and Aerospace Engineering Student Publications and Presentations

6-14-2019

\title{
A Multi-Fidelity Prediction of Aerodynamic and Sonic Boom Characteristics of the JAXA Wing Body
}

\author{
Forrest L. Carpenter \\ Texas A\&M University \\ Paul G. A. Cizmas \\ Texas A\&M University \\ Christian R. Bolander \\ Utah State University \\ Ted N. Giblette \\ Utah State University, tedngiblette@gmail.com \\ Doug F. Hunsaker \\ Utah State University, doug.hunsaker@gmail.com
}

Follow this and additional works at: https://digitalcommons.usu.edu/mae_stures

Part of the Aerospace Engineering Commons, and the Mechanical Engineering Commons

\section{Recommended Citation \\ Carpenter, Forrest L., Cizmas, Paul G. A., Bolander, Christian R., Giblette, Ted N., Hunsaker, Douglas F. "A Multi-Fidelity Prediction of Aerodynamic and Sonic Boom Characteristics of the JAXA Wing Body." AIAA Aviation 2019 Forum. Dallas, Texas, June 2019. https://doi.org/10.2514/6.2019-3237}

This Presentation is brought to you for free and open access by the Mechanical and Aerospace Engineering Student Research at DigitalCommons@USU. It has been accepted for inclusion in Mechanical and Aerospace Engineering Student Publications and Presentations by an authorized administrator of DigitalCommons@USU. For more information, please contact digitalcommons@usu.edu.

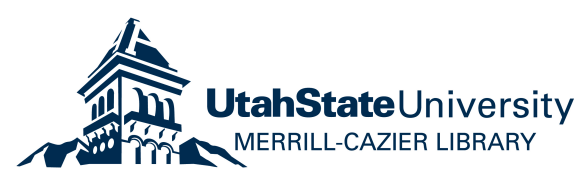




\title{
A Multi-Fidelity Prediction of Aerodynamic and Sonic Boom Characteristics of the JAXA Wing Body
}

\author{
Forrest L. Carpenter* and Paul G. A. Cizmas ${ }^{\dagger}$ \\ Texas A\&M University, College Station, TX, 77843-3141, USA \\ Christian R. Bolander ${ }^{\ddagger}$, Ted N. Giblette ${ }^{\S}$, and Douglas F. Hunsaker ${ }^{\mathbb{I}}$ \\ Utah State University, Logan, UT, 84322
}

\begin{abstract}
This paper presents a detailed comparison between the linear panel solver PANAIR A502 and the in-house Navier-Stokes solver UNS3D for a supersonic low-boom geometry. The high-fidelity flow solver was used to predict both the inviscid and laminar flow about the aircraft geometry. The JAXA wing body was selected as the supersonic low-boom geometry for this study. A comparison of the undertrack near-field pressure signatures showed good agreement between the three levels of model fidelity along the first $0.8 \mathrm{~L}$ of the signature. Large oscillations in the PANAIR results were observed. The PANAIR discrepancies were traced back to violations of the underlying assumptions within PANAIR: (1) small perturbation velocities and (2) no regions of transonic flow. These violations were due to large changes in surface curvature resulting in a strong expansion wave. While investigating the PANAIR discrepancy, measures of the fundamental assumptions of the Prandtl-Glauert equation used by PANAIR were quantified and used to assess the applicability of PANAIR to a given problem. Further comparison of surface temperatures predicted between the inviscid and laminar solutions was made. It was found that the recovery temperatures predicted by the inviscid solution were $5 \%$ less than those predicted by the laminar solution in likely candidate regions for distributed adaptivity. A surface deformation was added to the forward portion of the geometry to asses the viability of a future optimization study in this region. In this study, it was found that the near-field and ground signatures predicted by PANAIR and the UNS3D solutions responded in similar manners to the deformation.
\end{abstract}

Roman Letters

\section{Nomenclature}

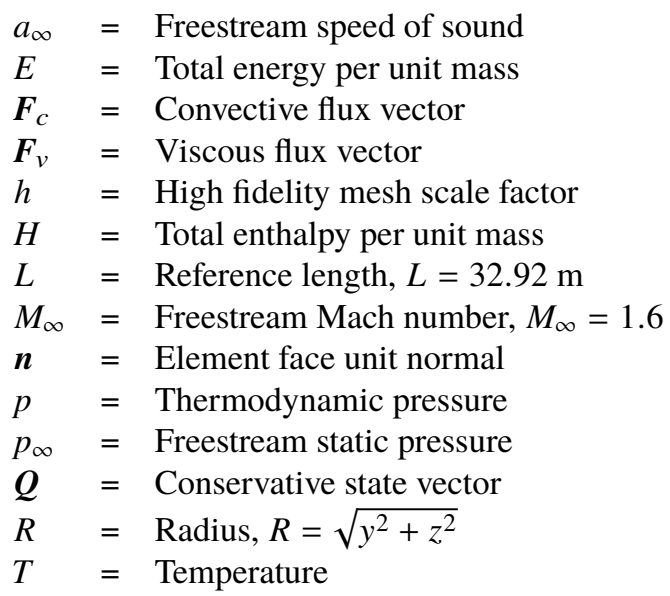

*Postdoctoral Associate, Department of Aerospace Engineering, 701 HR Bright Bldg, TAMU 3141, Member AIAA

${ }^{\dagger}$ Professor, Department of Aerospace Engineering, 701 HR Bright Bldg, TAMU 3141, Associate Fellow AIAA.

$¥$ Graduate Student, Mechanical and Aerospace Engineering Dept., 4130 Old Main Hill, AIAA Member.

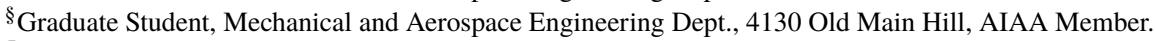

"IAssistant Professor, Mechanical and Aerospace Engineering Dept., 4130 Old Main Hill, AIAA Member. 
$v_{\mathbf{1}}=$ Perturbation velocity vector

$\boldsymbol{v} \quad=$ Velocity vector, $v=\{u, v, w\}^{T}$

$v_{\infty}=$ Freestream velocity vector

$V=$ Contravariant velocity

\section{Greek Letters}

$\delta_{i j}=$ Kronecker delta

$\mu \quad=$ Coefficient of viscosity of a Newtonian fluid

$\rho=$ Density

$\tau=$ Normalized distance along pressure sensor, $\tau=\left(x-R \sqrt{M_{\infty}^{2}-1}\right) / L$

$\phi=$ Perturbation velocity potential

$\Phi=$ Azimuth angle, $\Phi=\tan ^{-1}(y / z)$

$\Phi_{\infty}=$ Freestream velocity potential

\section{Introduction}

S UPERSONIC overland flight, and its commercialization, has become one of the driving forces in aerodynamics research

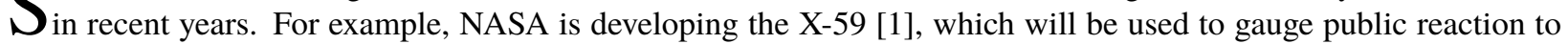
sonic booms and to help quantify appropriate standards for overland flight. In addition, a number of private companies have been pursuing efforts in designing supersonic business jets [2] and larger mid-sized supersonic transports [3, 4]. Each of these efforts represent a point design, meaning that the basis of the design is related to specific conditions to which the aircraft are subject. This means that deviations from the specified design conditions, whether related to the atmosphere, flight conditions, or maneuvers, could result in significant changes to the expected sonic boom loudness at ground level [5].

Distributed surface actuation represents one approach to mitigate the impact that the deviation from design conditions has on the sonic boom loudness. These distributed actuators modify the outer mold line of the aircraft to reshape the near-field flow, which creates a more favorable sonic boom loudness for the existing conditions. To reach this desired near-field signature, the size and location of the distributed actuators on the aircraft must be known. Due to the complexity of such a design space, it is beneficial to use a multi-fidelity framework where a lower-fidelity method explores the general design space and targeted studies and optimization is handled using high-fidelity solvers. The current work aims to provide a detailed comparison between the low-fidelity and high-fidelity tools that would be used in this type of multi-fidelity framework.

Previous work has investigated the use of high-order panel methods in the design of low-boom supersonic aircraft [6-8]. These efforts have shown that high-order panel methods can be used to accurately predict sonic boom loudness over large portions of the possible design space and have also highlighted a few geometries for which the panel solution falls short. In order to leverage the computationally inexpensive panel solutions, a multi-fidelity framework has been considered in the parametric optimization [9-11] of supersonic aircraft. A similar optimization strategy was suggested by Choi et al. [8] for the optimization of low-boom supersonic aircraft.

In a recent work, a sonic boom prediction framework was developed that used parametric geometry descriptions and panel solutions for the near-field flow [12]. The present work builds upon this effort and conducts a detailed comparison between the solutions given by panel and computational fluid dynamics (CFD) solutions. The CFD solver chosen for this work [13] has been most recently applied to a sensitivity analysis of a low-boom aircraft configuration [14]. This work also includes a validation case with a morphed geometry and identifies key metrics for assessing the suitability of a given geometry in the low-fidelity model.

\section{Methodology}

Predicting sonic boom loudness requires a description of the outer mold line (OML), an aerodynamic model, a sonic boom propagation tool, and a loudness metric. First, a description of the OML of the aircraft is generated. This OML is fed to an aerodynamic flow solver that is used to predict the near-field pressure signature of the aircraft. Then the near-field signature is propagated to the ground where the loudness is calculated. This section presents the methods used in each step of the loudness prediction process outlined above. First, the geometry description methodology is given, 
followed by descriptions of the two aerodynamic models considered, and of the propagation and loudness calculation tools used.

\section{A. Geometry Description}

Various methodologies have been developed for constructing a detailed description of the OML of supersonic transport geometries from a set of parametric surfaces [7, 12, 15]. The current work employs a multivariate spline technique with the low-fidelity tools to produce a representation of the chosen low-boom configuration.

\section{B. Aerodynamic Models}

Optimization studies require many data points to populate the design space and determine the "best" solution to a given problem. In general, the number of simulations needed to populate the design space and the long run times required by CFD prohibits the exclusive use of CFD for optimization problems. Lower fidelity aerodynamic tools provide sufficiently accurate physics to give a valuable solution with very short run times. As such, the lower fidelity tools can be used to quickly populate the design space and the CFD tools can be used to strategically enhance the database of optimal points. This section provides brief descriptions of both the low fidelity and high fidelity solvers used in this work.

\section{Low-Fidelity Model}

The low fidelity solver used in this work is PANAIR A502. PANAIR utilizes a high-order panel method to provide a linearized, inviscid description of subsonic and supersonic flows [16]. As a low-fidelity model, PANAIR has been used successfully in other efforts to evaluate the aerodynamic flow properties over supersonic aircraft. In addition, PANAIR is capable of determining the near-field pressure signature used for sonic boom prediction [6-8, 12].

The governing equations of PANAIR will be briefly covered in this section to provide context for the results shown later in this work. The purpose of this presentation is to identify key assumptions incorporated into the solution that PANAIR generates: therefore, many important details of the derivation will be ignored in the interest of identifying these assumptions. The full derivation of the governing equations can be seen in [17].

As stated previously, PANAIR provides an inviscid solution for both subsonic and supersonic flows. As such, its derivation begins with the Euler equations, which assumes that the flow is inviscid, steady, and free from the effects of body forces. Additionally, it is assumed that the flow is irrotational, isentropic, and that the fluid is an ideal gas. Irrotationality, in particular, is a requirement in all potential flow calculations and allows for the flow to be completely described by the velocity potential, $\Phi$, such that

$$
v=\nabla \Phi
$$

After applying each of these assumptions, the Euler equation can be transformed into the full potential equation [18], and then it can be linearized by defining a perturbation velocity potential and velocity such that $\phi=\Phi-\Phi_{\infty}$, and $\boldsymbol{v}=\boldsymbol{v}_{\infty}+\boldsymbol{v}_{\mathbf{1}}$. By assuming that the perturbation velocities are small,

$$
\left|v_{1}\right| \ll a_{\infty}
$$

where

$$
\left|v_{1}\right|=\frac{\left|v-v_{\infty}\right|}{\left|v_{\infty}\right|}
$$

and also assuming that the flow is neither transonic or hypersonic, meaning that

$$
M_{\infty}^{2}\left|v_{1}\right| \ll 1-M_{\infty}^{2}
$$

and

$$
M_{\infty}^{2}\left|v_{1}\right| \ll 1
$$

respectively, we can form the Prandtl-Glauert equation:

$$
\left(1-M_{\infty}^{2}\right) \phi_{x x}+\phi_{y y}+\phi_{z z}=0
$$

The Prandtl-Glauert equation can be further manipulated into an integral form which is the form of the equation that PANAIR discretizes to solve for the source and doublet distributions necessary to form a solution. 
It is important to note that, in supersonic flow, the resulting formulation corresponds to linear characteristics oriented at the free stream mach angle everywhere in the solution. As will be seen later on, this results in errors in shock orientation when the local velocity varies significantly from the freestream velocity.

The above derivation highlights the assumptions made to arrive at the Prandtl-Glauert equation which is a linear differential equation. As a consequence of assuming small perturbations (Eqs. (2)-(5)) only geometries that are long and thin and only purely subsonic/supersonic flows can be considered. Despite these restrictions, PANAIR has been used successfully in many applications as a low-fidelity aerodynamic model, as these assumptions are valid for a wide range of supersonic geometries and operating conditions.

\section{High-Fidelity Model}

The governing equations of the high-fidelity model used in this work are the non-linear mass, momentum, and energy conservation equations, collectively known as the Navier-Stokes equations. As such, the high-fidelity model is not hindered by the constraints used to reach the simplified panel method governing equations. The Navier-Stokes equations, written in integral form without any source terms, are given by

$$
\frac{\partial}{\partial t} \int_{\Omega} \boldsymbol{Q} d \Omega+\oint_{\partial \Omega}\left(\boldsymbol{F}_{c}-\boldsymbol{F}_{v}\right) d S=\mathbf{0}
$$

The conservative state vector, the convective flux vector, and viscous flux vector are given, respectively, by

$$
\boldsymbol{Q}=\left\{\begin{array}{c}
\rho \\
\rho u \\
\rho v \\
\rho w \\
\rho E
\end{array}\right\} \quad \boldsymbol{F}_{c}=\left\{\begin{array}{c}
\rho V \\
\rho u V+p n_{x} \\
\rho v V+p n_{y} \\
\rho w V+p n_{z} \\
\rho H V
\end{array}\right\} \quad \boldsymbol{F}_{v}=\left\{\begin{array}{c}
0 \\
n_{x} \tau_{x x}+n_{y} \tau_{x y}+n_{z} \tau_{x z} \\
n_{x} \tau_{y x}+n_{y} \tau_{y y}+n_{z} \tau_{y z} \\
n_{x} \tau_{z x}+n_{y} \tau_{z y}+n_{z} \tau_{z z} \\
n_{x} \Theta_{x}+n_{y} \Theta_{y}+n_{z} \Theta_{z}
\end{array}\right\}
$$

The components of the shear stress tensor are given, using Einstein notation, by

$$
\tau_{i j}=\mu\left(\frac{\partial v_{i}}{\partial x_{j}}+\frac{\partial v_{j}}{\partial x_{i}}-\frac{2}{3} \frac{\partial v_{k}}{\partial x_{k}} \delta_{i j}\right)
$$

The viscous work plus heat conduction term is

$$
\Theta_{\aleph}=v_{1} \tau_{\aleph x}+v_{2} \tau_{\aleph y}+v_{3} \tau_{\aleph z}+k \frac{\partial T}{\partial \aleph}
$$

where $\boldsymbol{\aleph}$ is a placeholder variable that can be substituted for by either $x, y$, or $z$.

The governing equations defined in Eq. (7) were solved using an in-house flow solver, UNS3D [13]. The flow solver was used to solve for both the inviscid and viscous flow fields around the aircraft, providing multiple levels of high fidelity solutions. Inviscid solutions were found by setting $\boldsymbol{F}_{v}=\mathbf{0}$ in Eq. (7). The viscous flow in this work was assumed to be laminar.

The flow solver uses an edge-based finite-volume framework to discretize Eq. (7). The convective fluxes are computed using Roe's approximate Riemann solver [19] with Harten's entropy fix [20]. A second-order spatially accurate solution was found by using piecewise-linear reconstruction to obtain the left and right states for the Riemann solver [21]. When computing the inviscid flows, a variation of the Venkatakrishnan limiter [22] was used to prevent nonphysical oscillations from occurring as a result of the piecewise-linear reconstruction. The Dervieux limiter [22] was used exclusively for the laminar simulation to provide the solution with additional dissipation and prevent divergence. Viscous fluxes were computed as a directed average at the quadrature point. Gradients of the flow were found using the least-squares with QR decomposition approach [23].

In this work, the flow around the aircraft was assumed to be steady. A steady solution of Eq. (7) was found by integrating forward in pseudo-time until the residual terms of the discretized equations were reduced by five orders of magnitude. The Generalized Minimal Residual Algorithm (GMRES) [24] was used as the primary method for time integration. A four-stage Runge-Kutta integration scheme [25] was used in the preliminary stages of the viscous simulations before switching to the GMRES algorithm. 


\section{Sonic Boom Propagation and Loudness}

The propagation of the near-field pressure signature to the ground was accomplished using the NASA tool called sBOOM. sBOOM solves the augmented Burgers' equation to propagate the near-field signature along ray tubes to the ground [26]. The algorithm implemented in SBOOM includes the effects of molecular relaxation and thermoviscous absorption [26-29]. The use of this tool in the current work is motivated by its ability to accurately model rise times, which have been shown to directly affect the loudness of a given pressure signature. For comparison with data found in the literature, the US Standard Atmosphere [30] was used in conjunction with the default humidity profile and no specified wind profile.

The loudness metric of choice for this work was Stevens' perceived level of loudness [31] measured in decibels (PLdB) and implemented using Stevens' Mark VII algorithm. The perceived level metric has been shown to best approximate the human perception of various sounds, including sonic booms [31-33]. Two computational tools were used to compute the PLdB values of the sonic booms encountered in this work. The first was the well established NASA tool called Loudness Code for Asymmetric Sonic Booms (LCASB) [34]. The second was a newly developed, open-source tool known as PyLdB [35]. Good agreement has been seen between both LCASB and PyLdB for a number of ground signatures [35]. Even so, both tools were used in this work for completeness and to provide a point of further comparison between the two tools.

\section{PANAIR A502 Applicability Using Fundamental Assumptions}

It is possible that PANAIR could be found to be an unsuitable lower fidelity aerodynamic solver for certain cases as a result of the underlying assumptions stated previously in Sec. III.B.1 As such, a case that might pass the "eye test", i.e., would not be expected to violate PANAIR's assumptions, could indeed fail to satisfy the underlying assumptions. A solution from a higher fidelity solver, such as an Euler solution, could be used to evaluate PANAIR's applicability for a given case. An analysis of the perturbation velocity on the surface can highlight regions of the body where the PANAIR solution will likely yield both poor surface and near-field pressures.

An analysis of the local Mach number on the surface of a geometry is generally enough to identify regions where the assumptions made by PANAIR in regards to transonic and hypersonic flow will start to be violated. However, the assumption that the flow will not enter the transonic regime can also be checked by rearranging Eq. (4) such that

$$
\left|\frac{M_{\infty}^{2}\left|\boldsymbol{v}_{\mathbf{1}}\right|}{1-M_{\infty}^{2}}\right| \ll 1
$$

Note that the inclusion of the absolute value in Eq. (8) is a result of changing Eq. (4) to be valid for supersonic flows. A similar evaluation can be done using Eq. (5) to indicate the presence of regions with large perturbation velocities or supersonic Mach numbers in the high-fidelity solution. Analyzing the perturbation velocity along with these indicators on a geometry allows for a preliminary identification of problem areas in the PANAIR solution.

\section{Case Description}

The low-boom case selected for this work was the JAXA Wing Body (JWB) geometry, which is shown in Fig. 1 . The JWB was designed to match the under-track equivalent area distribution of the NASA Configuration 25D low-boom concept aircraft [36]. This low-boom geometry was developed specifically for the Second AIAA Sonic Boom Prediction Workshop and was one of the required cases in the workshop [37]. In keeping with Park and Nemec [37], the reference length used herein is $32.92 \mathrm{~m}$ as opposed to the length of the JWB, which is $38.7 \mathrm{~m}$.

\section{A. Low-Fidelity Computational Domain Description}

Using a multivariate interpolation of the outer mold line given by the CFD surface mesh, a structured surface mesh was created for PANAIR. The panels on the wing were cosine-clustered in both the span-wise and chord-wise direction with the clustering occurring on the leading and trailing edges as well as the wing tip, but not at the wing root. The wing tip was capped with a flat network consisting of the same number of chord-wise panels as the outboard section of the wing surface with 5 panels in the vertical direction. The fuselage was cosine-clustered along the tail of the fuselage in both the axial and azimuthal directions to attempt to resolve the sharp corner along the edge. The nose of the fuselage was cosine-clustered in the axial direction near the nose and the mid-body shared its axial paneling with the wing root. Both of these sections shared the azimuthal paneling with the tail. 
Fig. 1 Rendering of the supersonic low-boom JAXA Wing Body geometry.

5.38

A panel refinement study, which will be discussed in Sec. V] was used to determine the appropriate number and clustering of the panels to achieve a converged surface pressure field. Figure 2 shows the final PANAIR surface mesh used for the baseline JWB geometry with 3,650 panels on the wing and 3,950 panels on the fuselage. Also shown in Fig. 2 are the PANAIR boundary conditions used as inputs, which are necessary to run the geometry in PANAIR. A description of these boundary conditions are given in the PANAIR User's Manual [38].

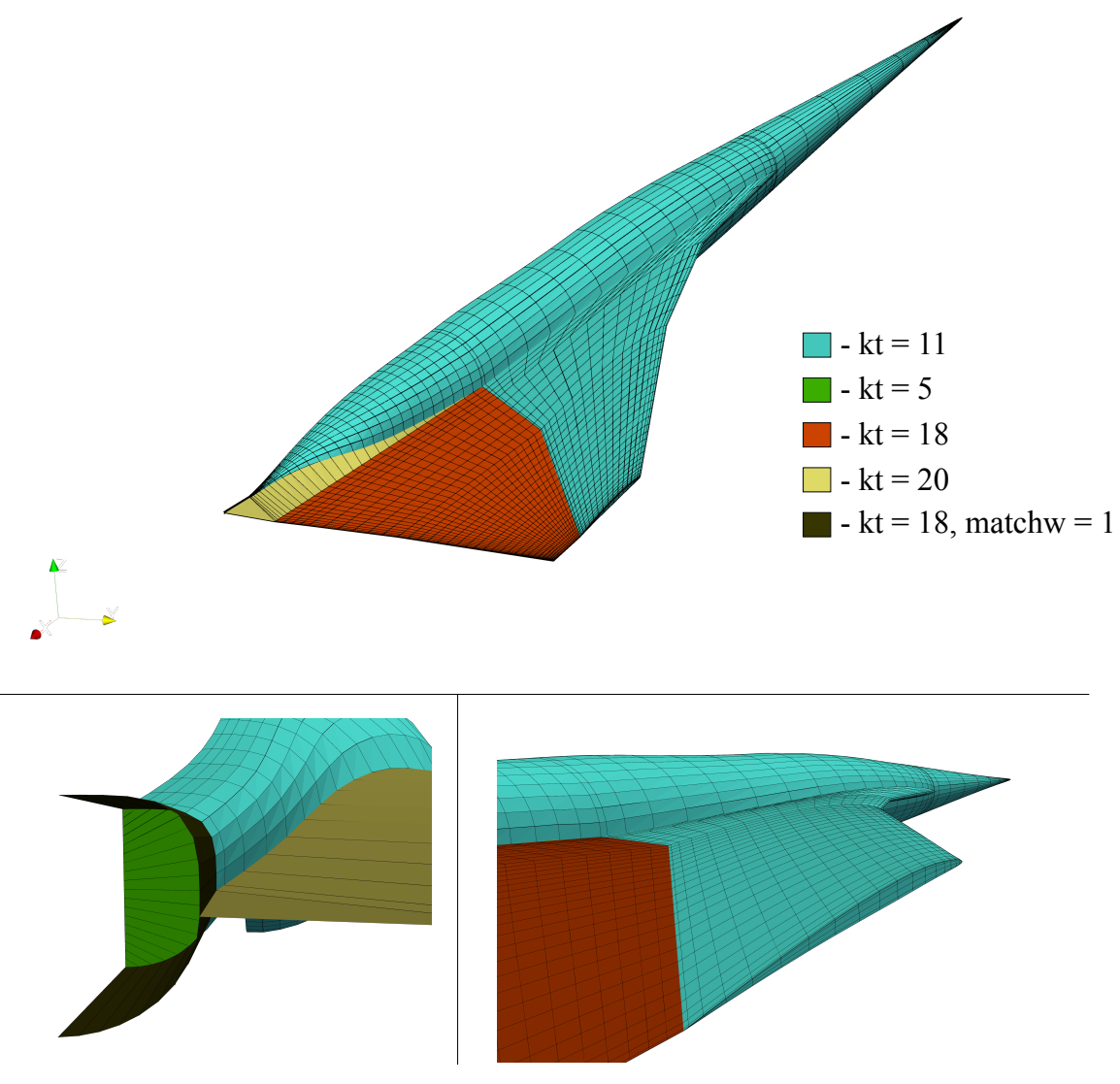

Fig. 2 Illustration of the final PANAIR surface networks with 7,600 panels and the applied PANAIR boundary conditions for the JWB geometry.

\section{B. High-Fidelity Computational Domain Description}

The prediction of both inviscid and viscous flows necessitated two different computational mesh topologies be used in this work. For inviscid flows, a set of three uniformly refined tetrahedral meshes were used. A single unstructured 
mixed element mesh was used for the viscous prediction. Table 1 presents the dimensions of all four meshes used, and gives the mesh scale factor for each grid. Following Park and Nemec [37], the mesh scale factor was defined as $h=\left(10^{7} / N_{\text {node }}\right)^{1 / 3}$.

Table 1 High-fidelity computational mesh dimensions.

\begin{tabular}{rrrrrrr}
\hline Flow & $h$ & Nodes & Tetrahedra & Pyramids & Prisms & Hexahedra \\
\hline Inviscid & 1.15 & $6,491,425$ & $37,397,159$ & 0 & 0 & 0 \\
Inviscid & 0.96 & $11,335,260$ & $65,432,421$ & 0 & 0 & 0 \\
Inviscid & 0.81 & $18,875,613$ & $109,141,197$ & 0 & 0 & 0 \\
Viscous & 0.85 & $16,267,427$ & $13,741,489$ & 530,328 & $1,326,858$ & $12,838,541$ \\
\hline
\end{tabular}

Figure 3 aillustrates the difference in the physical sizes of the two different computational mesh topologies. The inviscid mesh had a much larger domain size than the viscous mesh, extending over twice the distance in some directions. Figure $3 \mathrm{~b}$ shows the difference in the surface grid topology between the inviscid and viscous grids. The viscous surface grid was made up primarily of unstructured quadrilaterals; whereas, the inviscid surface mesh was tessellated purely with triangles. The viscous mesh was required to capture the boundary layers adjacent to every wall surface. To accommodate this, a boundary layer mesh was created by extruding the surface mesh along mesh normals using a boundary layer growth rate of 1.118 , with a first cell height of $6.096 \times 10^{-3} \mathrm{~mm}$. All of the computational meshes used in this work were the same as those prepared for the Second AIAA Sonic Boom Prediction Workshop [37].

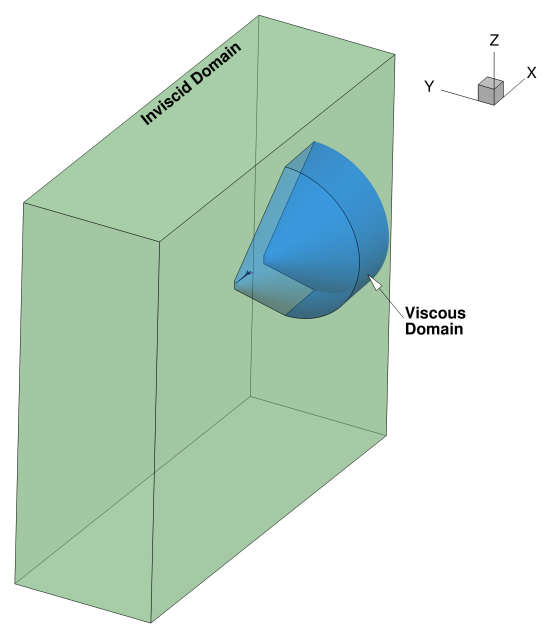

(a) Computational Domain Comparison.

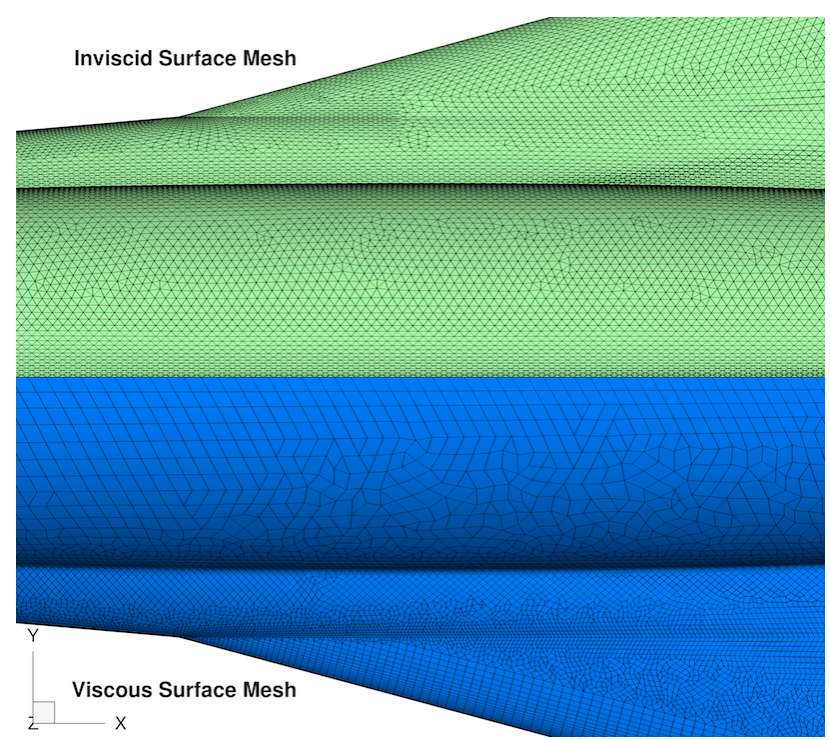

(b) Surface Mesh Comparison.

Fig. 3 Comparison of the workshop inviscid computational mesh (green) and the workshop viscous computational mesh (blue). The "medium" inviscid mesh is being shown.

\section{PANAIR A502 Panel Refinement Study}

Preliminary results for the JWB showed discrepancies between the Euler and PANAIR solutions on the outboard section of the wing as well as on the tail. A grid convergence study was performed on both the wing and fuselage to ensure proper paneling and a fully resolved solution from PANAIR. The pressure distributions over both the top and bottom of the wing were compared at span-wise stations of $2 y / b=0.23,0.4,0.5,0.7,0.85$, and 0.95 , with 20 cosine-spaced panels in the chord-wise direction. Each of the wing sections were subdivided into a certain number of 
span-wise panels as shown in Table 2 .

Table 2 Number of wing section panels in the span-wise direction used in the grid convergence study.

\begin{tabular}{crrr}
\hline \multirow{2}{*}{ Mesh } & \multicolumn{3}{c}{ Wing Sections } \\
& Inner & Middle & Outer \\
\cline { 2 - 4 } Coarse & 2 & 3 & 8 \\
Medium & 4 & 6 & 16 \\
Fine & 8 & 12 & 32 \\
\hline
\end{tabular}

The results of the span-wise grid convergence study show that, over the range of panels used in this study, the wing is insensitive to paneling in the span-wise direction except at the tip of the wing. This is to be expected, as the gradients in the flow will be strongest in the free-stream direction of the flow and the geometry is smooth in the span-wise direction.

The chord-wise grid convergence study of the wing was conducted using a constant span-wise paneling of the medium level as specified in Table 2. The coarse, medium, and fine chord-wise convergence cases were constructed using 10, 20, and 40 cosine-spaced panels respectively. The solution is much more sensitive to paneling in the chord-wise direction and so the number of panels in the "fine" level of refinement was used to mesh the JWB in PANAIR.

A grid convergence study for the fuselage was also conducted. Since the nose of the aircraft is nearly axisymmetric, a grid can be used in PANAIR similar to that used by Giblette and Hunsaker [12], where the azimuthal distribution of panels were most important. In fact, in the axial direction, as little as 14 panels that were cosine-clustered near the tip were sufficient to provide a converged solution on the nose.

The results of the grid convergence study on the tail, however, were inconclusive and showed some interesting behaviors, such as the oscillations in Figure 4. This phenomenon will be discussed in greater detail as the surface pressure solutions are discussed later in this work. Using a fairly coarse paneling on the tail in the axial direction avoided the ringing in the solution, so the same coarsening algorithm used by Giblette and Hunsaker [12] was used to refine the tail paneling. The section of the fuselage that connected to the wing shares the same paneling as the wing root in the chord-wise direction and 30 panels around the circumference.

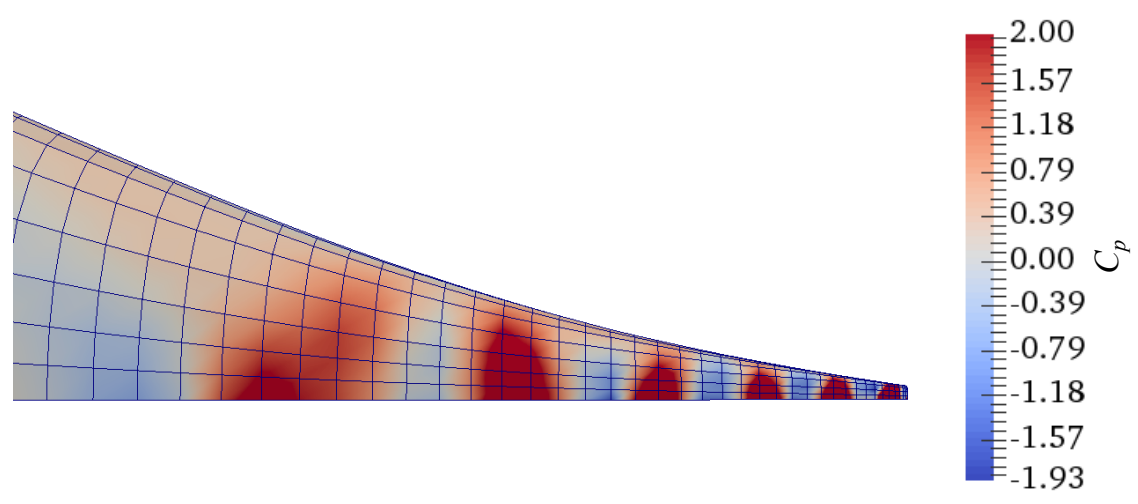

Fig. 4 Oscillations in the PANAIR pressure solution as the grid is refined.

The final grid used in PANAIR, as shown in Fig. 2, exhibits the computational benefit to using this low-fidelity tool as part of a multi-fidelity framework. The number of panels used in this solution is close to the maximum allowable panels that can be used in the version of PANAIR used. After passing the mesh into PANAIR, running the case took approximately 90 seconds, which lends well to the design space exploration needed to inform a high-fidelity model.

\section{Comparison of Low-Fidelity and High-Fidelity Model Results}

This section presents the comparison of the low-fidelity and high-fidelity model results. Comparison to data from the Second AIAA Sonic Boom Prediction workshop is made where possible. The converge characteristics of the high-fidelity flow solver are presented first. The solver convergence is followed by a discussion of the near-field and sonic boom prediction results. The PANAIR surface results are then presented in detail, and the applicability of PANAIR with 
the JAXA wing body case is discussed. Finally, a comparison of the high-fidelity surface temperature predictions is given.

\section{A. High-Fidelity Model Convergence}

The primary solution criterion used in this work was defined to be a five-order reduction in the solution residual magnitudes. A reduction of five-orders proved to be sufficient to achieve a converged value of the sonic boom loudness. Figure 5a shows the convergence histories for the Euler solutions on the three inviscid grids as well as for the laminar solution. The Euler solutions were all observed to converge in less than 3,000 iterations, taking at most seven hours for the finest grid ${ }^{*}$ The convergence of the laminar solution residuals was observed to stall after roughly 3,000 iterations. Due to the stalled solution residuals, the history of the integrated forces were used to judge convergence. In this case, convergence was declared once the forces appeared to stop changing significantly. The force histories for the different cases are shown in Fig. 5b. It took 25,000 iterations for the average variation of the viscous forces to approach a constant value.

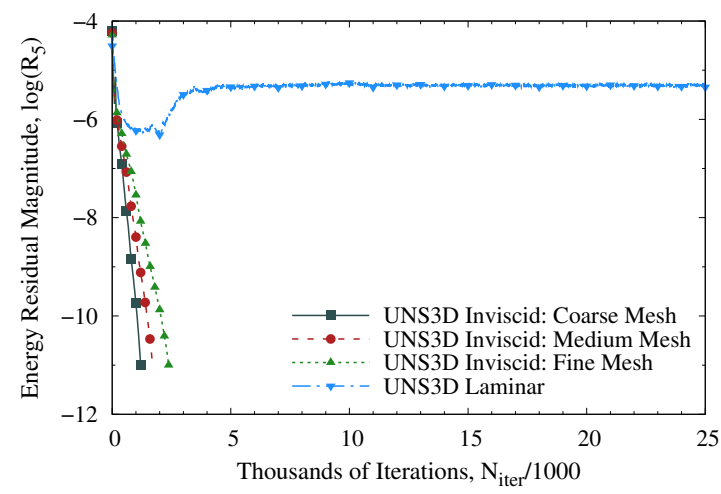

(a) Residual History

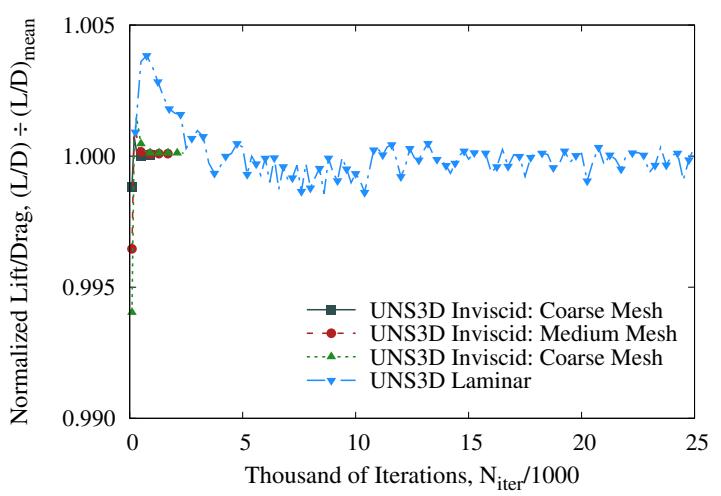

(b) Force History

Fig. 5 Residual and force convergence histories for the high fidelity solver solutions. Mean $L / D$ values for coarse, medium, fine Euler and laminar were 11.07, 11.08, 11.09, and 9.51, respectively.

\section{B. Aerodynamic and Sonic Boom Metric Comparison}

Figure 6 shows near-field pressure signatures extracted at $R / L=3$ and $\Phi=0^{\circ}$ from the PANAIR solution, the Euler prediction using the finest mesh, and from the laminar prediction. Relatively good agreement between all three models was observed for the first $80 \%$ of the pressure signature. Beyond this point the three models begin to deviate from one another. Large pressure fluctuations were seen in the PANAIR signature that are not present in either high-fidelity signatures. The signature from the laminar prediction showed a much more severe and delayed re-compression of the near-field pressure at approximately $\tau=1.1$. This difference in the inviscid and laminar solutions was due to flow separation at the tail of the aircraft, which was not present in the inviscid solution.

The large pressure fluctuations observed in the PANAIR prediction were a cause for concern, as fluctuations of this magnitude will force the sonic boom loudness to increase dramatically. A statement from Epton and Magnus [17], in an outline of the theory of PANAIR, provides insight into the likely culprit:

"In exact theory, the expansion waves are in the form of a continuous centered fan of characteristics instead of a single discontinuity. The approximation of expansion waves by an expansion shock is valid near the surface but is a poor representation of the flow at greater distances away from the surface. Linearized theory solutions involving rapid expansions can be expected to yield good pressure distributions on the surface but the induced flow at large distances from the expansion surface will not be accurately described."

*Fine Euler solution computed using 504 Intel Xeon E5-2680 v4 2.4GHz processors. 


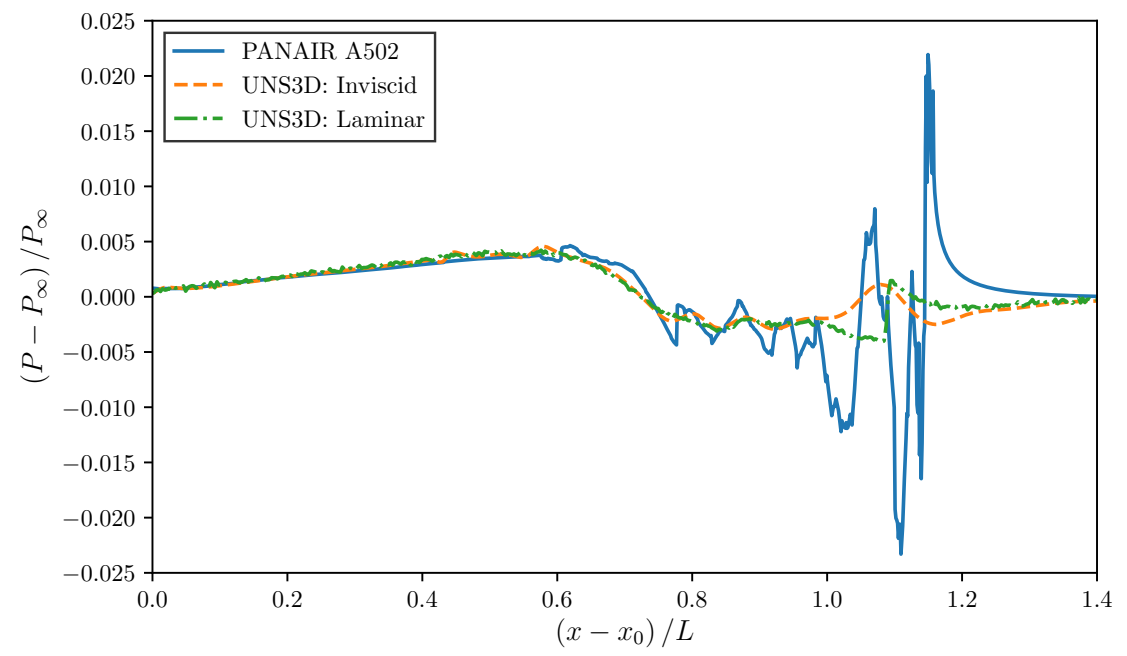

Fig. 6 Comparison of the near-field signatures for the JWB.

From this statement we learn that, in regions of strong expansion, accurate surface pressures may be achieved, but as the solution is taken at distances far from the surface, the results will be poor. The surface pressures predicted by PANAIR in this work are examined in more detail in a following subsection.

Figure 7 shows the ground signatures of the propagated near-field pressure signatures, and their respective PLdB values are shown in Table 3. The linearity of the PANAIR solution was evident in the ground signatures, as signature peak is shifted to the right slightly from the non-linear solutions. This is a result of not accounting for the non-linear aging of the shocks in the PANAIR solution. As expected, the PANAIR solution predicted the loudest sonic boom signature of the three models. The sharp, delayed re-compression observed in the near-field signature of the laminar prediction was partially preserved by the ground signature, reducing the pressure expansion and giving the quietest sonic boom. Figure 8a shows that the PLdB values for all of the UNS3D inviscid predictions were in excellent agreement with the inviscid predictions submitted to the Second AIAA Sonic Boom Prediction Workshop (SBPW2) [37].

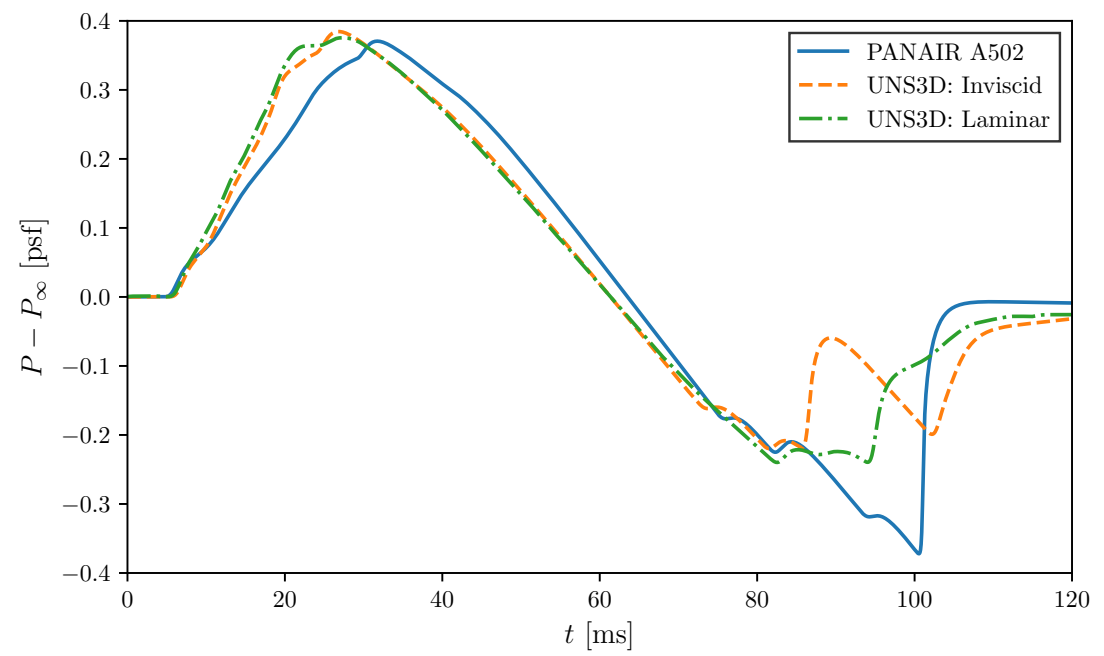

Fig. 7 Comparison of the ground signatures for the JWB.

Figures $8 \mathrm{~b}$-d compare the lift, drag, and pitching moment coefficients for each aerodynamic model tested against 
Table 3 Comparison of perceived level in decibels for PANAIR, the UNS3D Euler solution, and the UNS3D Laminar solution.

\begin{tabular}{lrrr}
\hline \multirow{2}{*}{ Method } & \multicolumn{3}{c}{ Model Fidelity } \\
& PANAIR A502 & Fine Inviscid & Laminar \\
\cline { 2 - 4 } PyLdB & 86.53 & 79.93 & 75.79 \\
LCASB & 86.54 & 79.98 & 75.74 \\
\hline
\end{tabular}

themselves and the inviscid coefficient values submitted to the workshop. The current inviscid predictions were found to be in excellent agreement with the mean workshop values. The PANAIR-predicted aerodynamic coefficients were reasonably similar to the fine mesh. PANAIR was found to under-predict the drag coefficient by $5.8 \%$ and over-predicted the lift and pitching moment coefficients by $1.6 \%$ and $0.9 \%$ respectively. As expected, the laminar solution showed a marked increase in drag (11.54\% difference from the fine mesh), and also a $4.3 \%$ decrease in lift from the fine inviscid case.

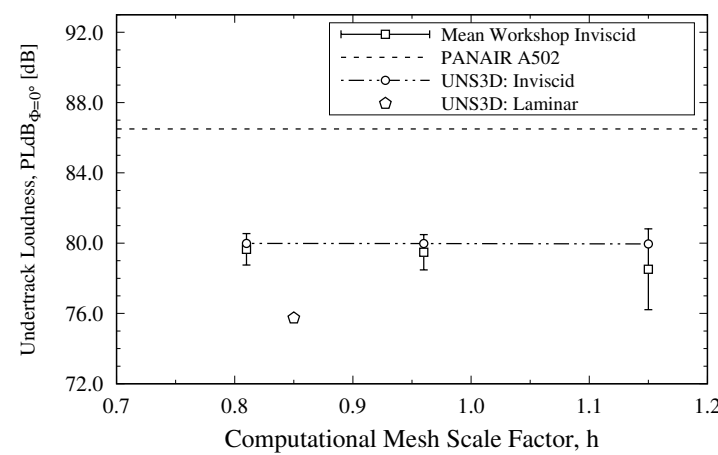

(a) Undertrack Sonic Boom Loudness

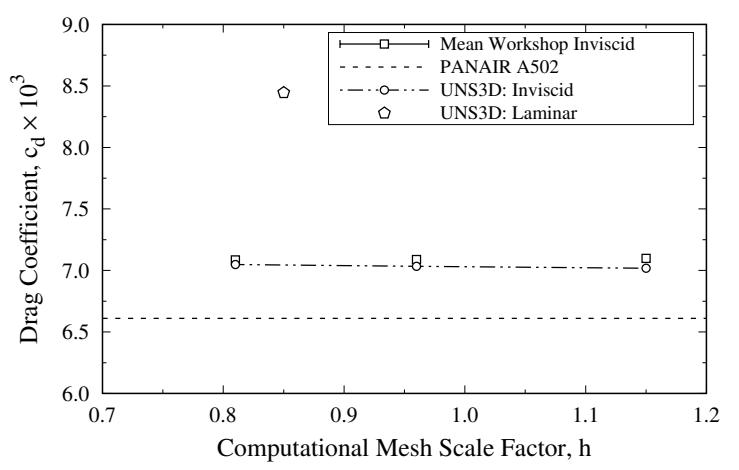

(c) Drag Coefficient

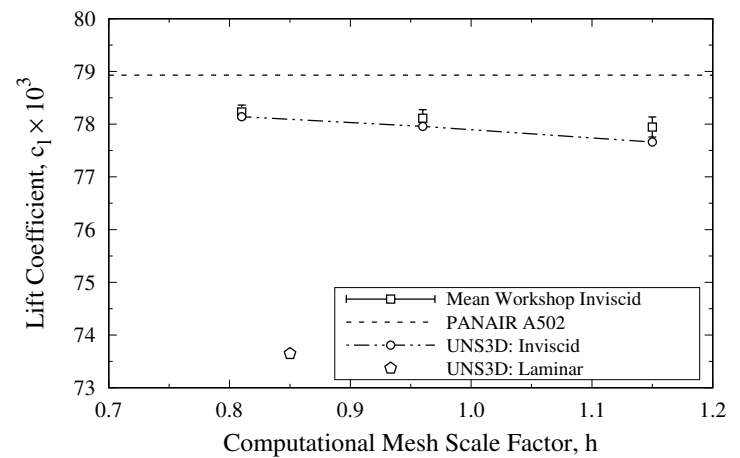

(b) Lift Coefficient

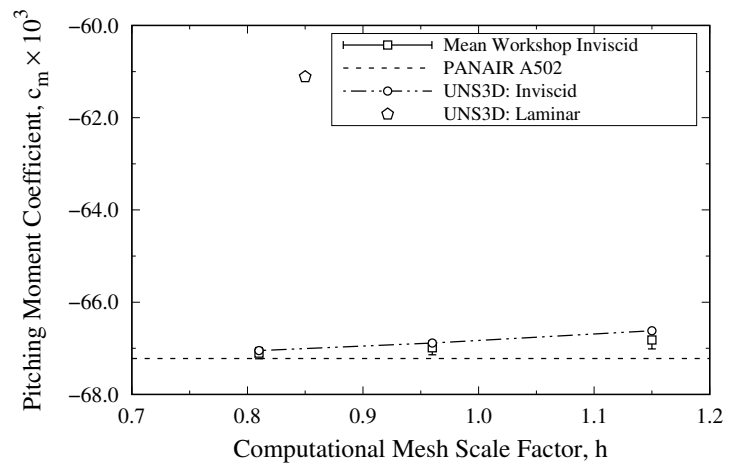

(d) Pitching Moment Coefficient

Fig. 8 Comparing current predictions of forces, pitching moment, and undertrack loudness using all levels of model fidelity against SBPW2 data.

\section{Surface Pressure Comparison}

A comparison between the PANAIR and UNS3D inviscid surface pressures for the top and bottom surfaces of the aircraft is shown in Figs. 9 and 10. These figures show that, qualitatively, the surface solutions match well for the majority of the geometry. Further insight can be gained by examining the perturbation velocities, defined by Eq. (3), using the UNS3D inviscid solution. Examining the perturbation velocities helps to identify areas of the solution that will not be represented well by the fundamental equations used by PANAIR that were described in Sec. III.B.1. Figures 


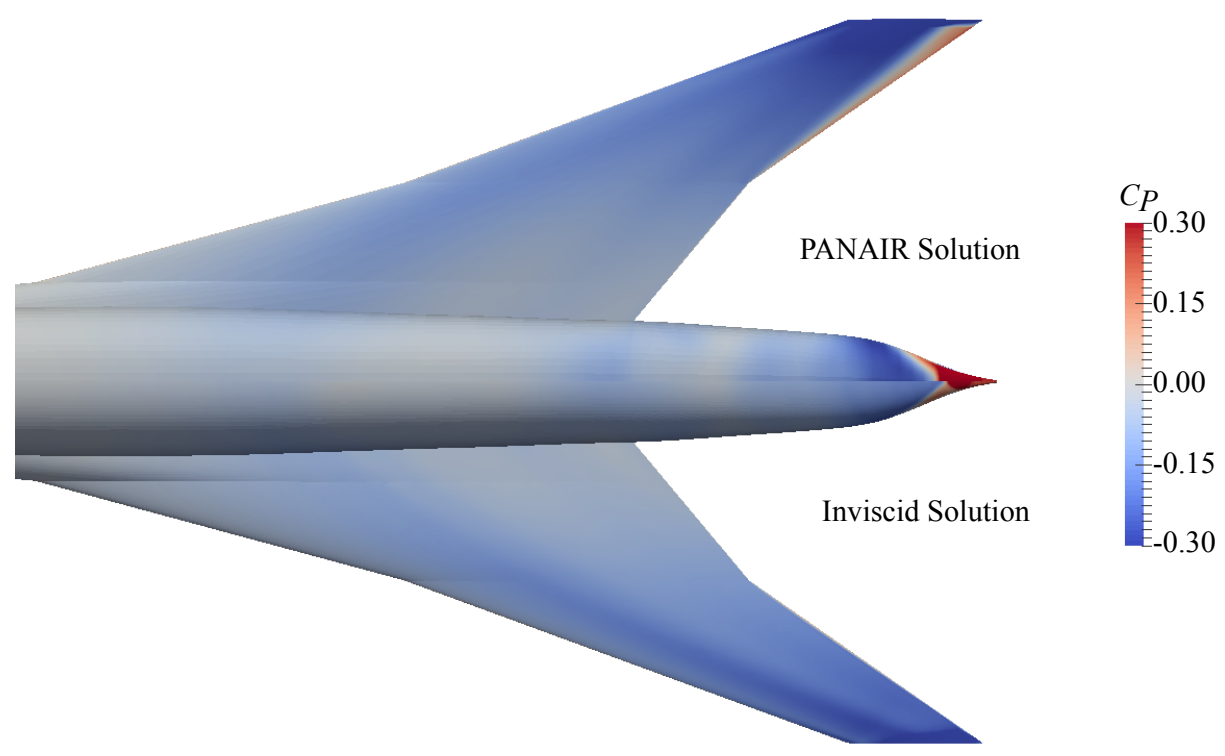

Fig. 9 Comparison of PANAIR and UNS3D inviscid surface pressures for the top surface of the JWB.

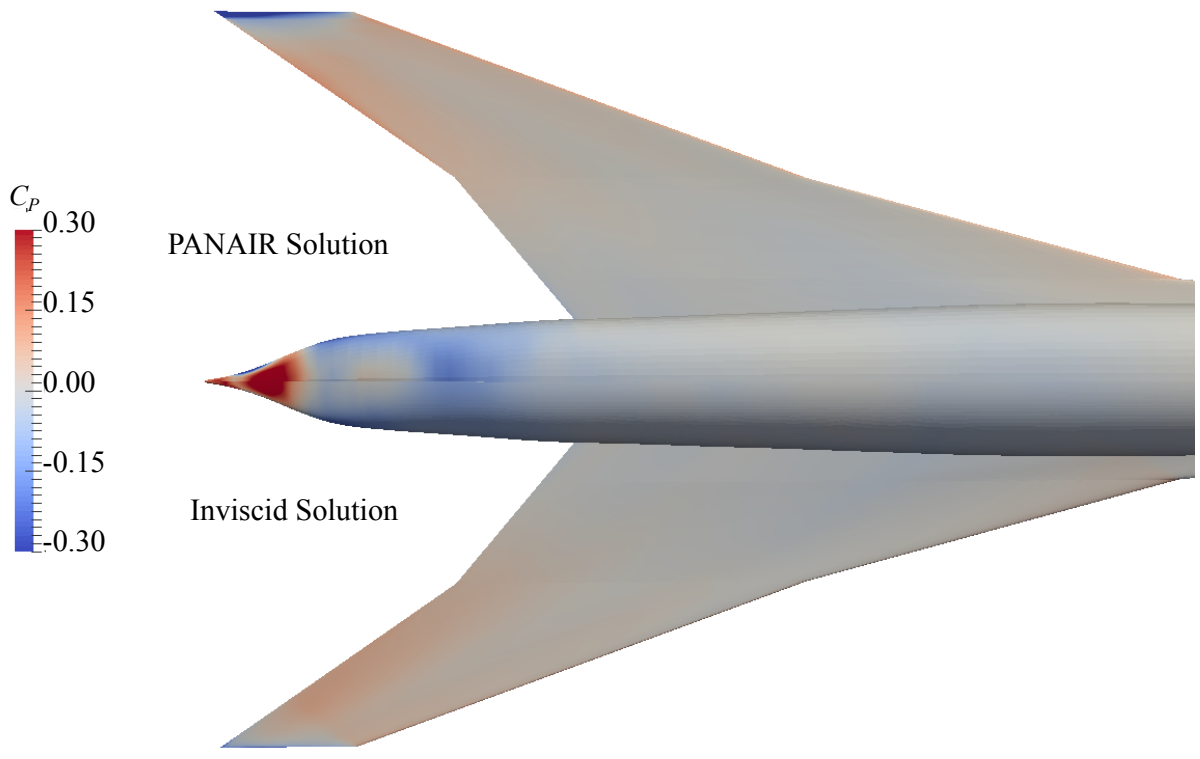

Fig. 10 Comparison of PANAIR and UNS3D inviscid surface pressures for the bottom surface of the JWB.

11 and 12 show the normalized perturbation velocities for the top surface of the wing and both surfaces of the fuselage, where the perturbation velocity was normalized by the freestream velocity.

Figures 9 and 10 indicate that there are discrepancies between the UNS3D and PANAIR solutions at the tail and outboard sections of the wing. Those areas correspond to the same areas in Fig. 11 and Fig. 12 where the normalized perturbation velocity is, in many regions, much greater than the freestream Mach number, which violates Eq. (2). Due to the relative orientation of these regions of the wing and fuselage and the corresponding freestream Mach angle, these areas are actually independent of one another and can be inspected individually. 


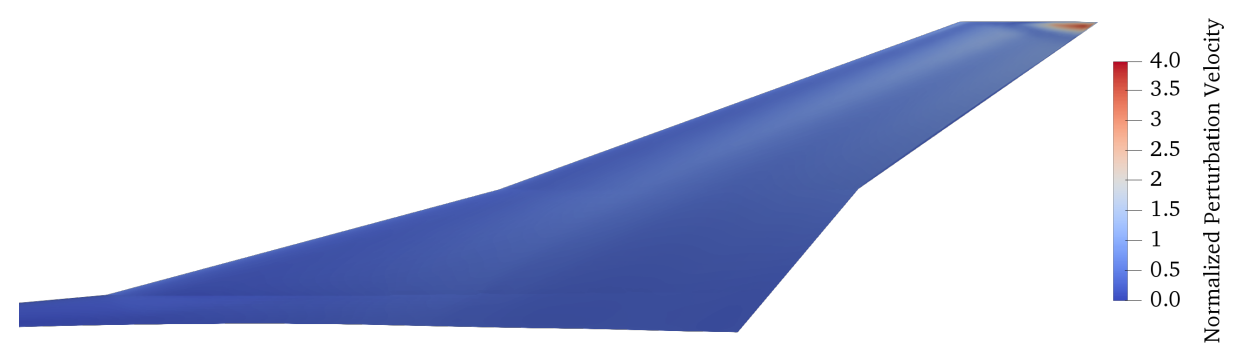

Fig. 11 Normalized perturbation velocities for the upper surface of the JWB wing.

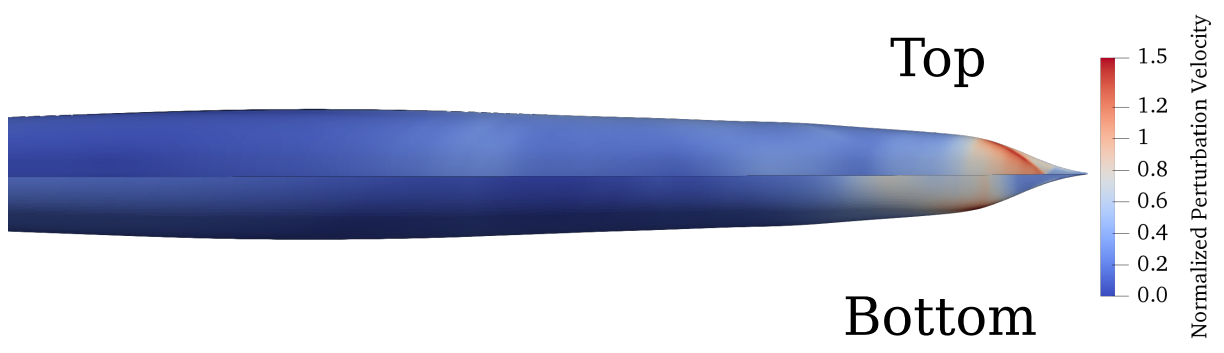

Fig. 12 Normalized perturbation velocities for the fuselage of the JWB.

\section{Analysis of PANAIR Solution on the Wing}

The outboard portion of the wing has noticeable differences on the trailing edge as well as the wing tip. Issues with the wing tip are to be expected in a potential flow solver such as PANAIR, because an infinite velocity is predicted around sharp corners. However, the increased grid refinement and cosine clustering performed in the span-wise direction can limit the impact of this discrepancy to the very edge of the wing, thus reducing the adverse impact on the rest of the solution. It is expected that these velocity spikes on the wing tip could be further mitigated by creating rounded corners on the wing tip with a dense surface mesh resolution.

The pressure on the trailing edge of the outboard section of the top of the wing represents the pressure changes that would be seen in the presence of a shock wave, though this shock is not present in the Euler solution. This is due to the use of linear characteristics in PANAIR that are inclined at the freestream Mach angle throughout the solution. The shock shown in the PANAIR solution is therefore inclined at the freestream Mach angle, though the local Mach number in this region is higher and would create a supersonic trailing edge.

\section{Analysis of PANAIR Solution on the Fuselage}

Figure 12 shows that the area of high curvature on the tail of the aircraft is an area of potential concern. These areas are likely to test the limits of the linearized supersonic solution given by PANAIR. Since the pressure distribution indicates that there is a shock on the tail, the geometry can be tested against Eq. (8) to check how well this region meets the assumptions of PANAIR in regards to transonic flow. The results of that test on the Euler solution are shown in Fig. 13 and indicate that this region is indeed violating the assumption of no transonic flow and, in fact, the Euler solution gives Mach numbers below unity on part of the surface of the tail.

Figure 12 also shows a region on the side of the fuselage where the perturbation velocities are large. This is an area where cross-flow is likely to occur from the bottom to the top of the tail due to the relatively sharp edge present in the geometry. The combination of the cross-flow and the sharp edge create an increase in the perturbation velocity that violates the linearized assumptions of PANAIR. 


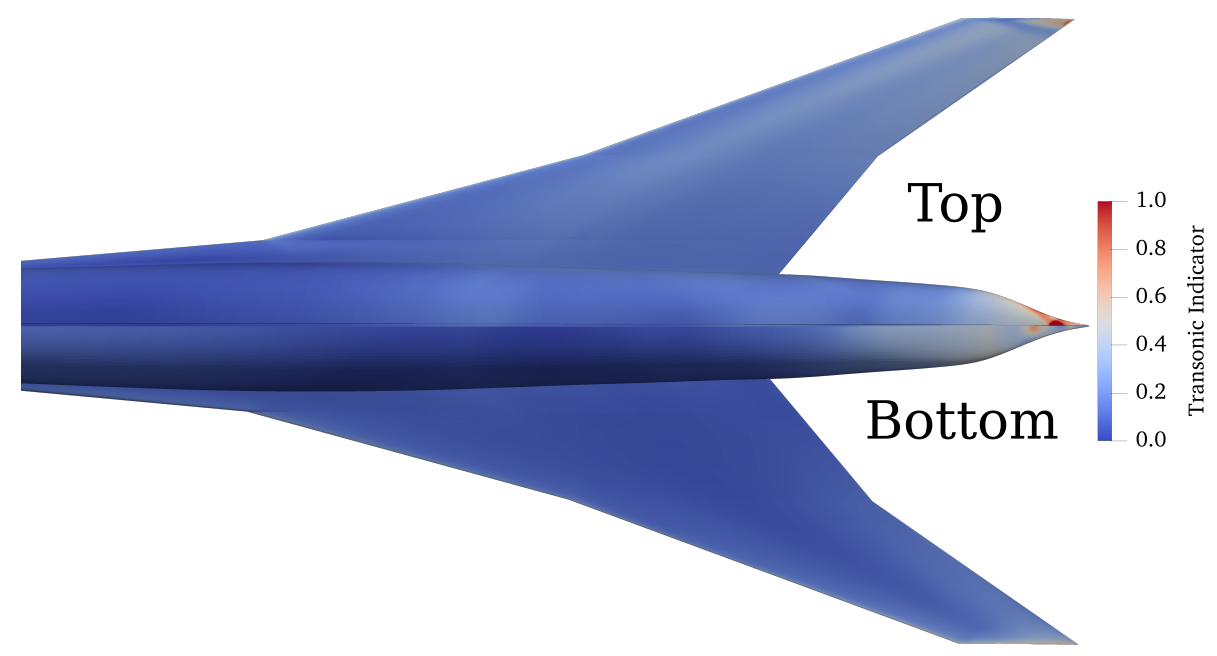

Fig. 13 PANAIR transonic indicator over the tail section of the JWB.

On the bottom of the fuselage there are some areas of increased perturbation velocity similar to the top surface. The shock on the bottom is weaker than on the top, however, and the Mach numbers are not as close to the transonic regime. This explains why the PANAIR results shown in Fig. 10 do not deviate as drastically on the bottom of the aircraft as on top, though the small differences between the two solutions are likely a result of the relative magnitude of the perturbation velocities.

In summary, the large spikes seen at the rear of the near-field signature correspond to areas on the JWB where the assumptions made by PANAIR are not met. Specifically, the tip of the tail enters a transonic regime, which explains the oscillations observed during the grid convergence study. In addition, perturbations velocities on the order of the freestream velocity on certain sections of the wing and tail degrade the solution further. The first $75-80 \%$ of the aircraft gives results that are in good agreement with the Euler solution and may provide an area where modifications to the geometry can be accurately represented.

\section{High-Fidelity Surface Temperature Comparison}

Accurate predictions of aircraft surface temperatures are important for the material selection process for the distributed actuator devices. The actuator designer requires an accurate range of temperatures that the actuator would encounter during normal operation. Due to the long simulation run times associated with viscous flows, it would be ideal if the inviscid solution could provide a sufficiently accurate alternative.

For an inviscid solution, the temperature at the wall can be found using the equation for the temperature recovery factor, given by [39]

$$
r=\frac{T_{R}-T_{\ell}}{T_{0, \ell}-T_{\ell}}
$$

where $T_{R}$ is the recovery temperature, $T_{\ell}$ is the local temperature, and $T_{0, \ell}$ is the local stagnation temperature. It has been found for laminar flows that $r \approx \sqrt{P r}$. Using the equation for stagnation enthalpy and rearranging Eq. (9), one can arrive at the recovery temperature, i.e., the temperature at the wall, as a function of the local Mach number and the local temperature:

$$
T_{R}=T_{\ell}\left(1+\sqrt{P r} \frac{\gamma-1}{2} M_{\ell}^{2}\right)
$$

The recovery temperatures on the upper surface of the geometry were found to be nearly uniform, as shown in Fig. 14a. Only at the tips of the wings and just upstream of the tail of the aircraft are slight variations in the contours observed. By contrast, the laminar surface temperatures, shown in Fig. 14b indicate strong flow features along the upper surface of the wings and in the tail region. Strong expansion waves along the tail resulted in a region of high surface temperatures. Figure $14 \mathrm{c}$ shows the percent difference, defined as $d_{r}\left(T, T_{R}\right)=T_{R} / T-1$, in the two surface 
predictions. It was found that in regions favorable for placement (body center-line and the inner area of the wings), the difference between the Euler and laminar predictions was on the order of $-5 \%$. The notable difference on the tail where the relative difference between the solutions is on the order of $-30 \%$.

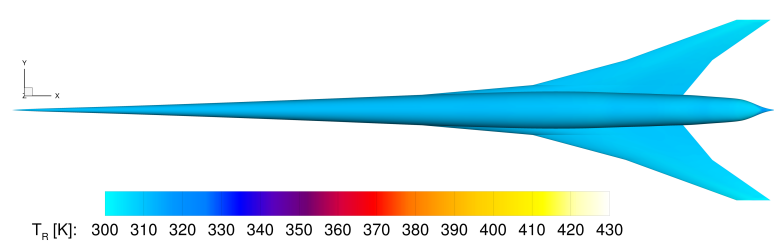

(a) Inviscid: Surface Recovery Temperatures

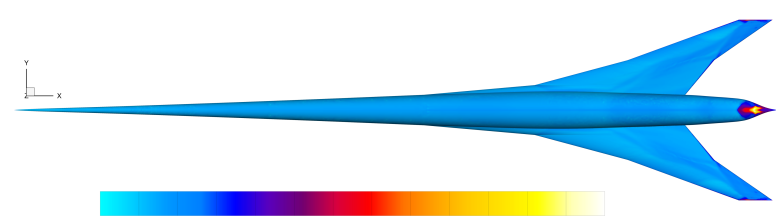

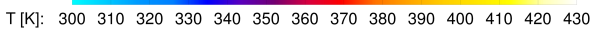

(b) Laminar: Surface Temperatures

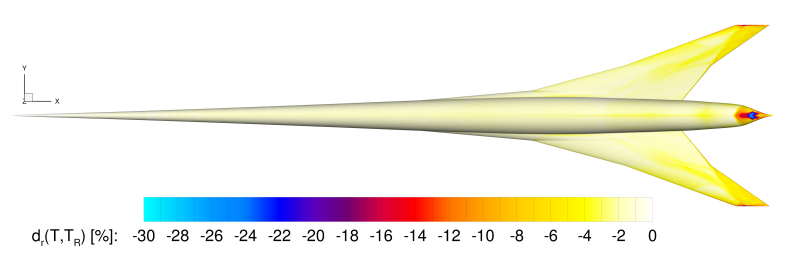

(c) Surface Temperature Percent Differences

Fig. 14 Comparing surface temperatures predicted using the medium mesh inviscid solution with laminar surface temperatures on the aircraft upper surface.

Figure 15a shows the lower surface recovery temperatures, and like on the upper surface, the temperatures were found to be nearly uniform. The lower surface laminar surface temperatures are more uniform than those on the upper surface, as shown in Fig. 15b. The notable exception being a large region of high surface temperatures just upstream of the tail, again due to a strong local shock feature. Avoiding the regions adjacent to the wing tips and the tail, the difference between the inviscid and laminar surface temperatures was again on the order of $-5 \%$. The hot spot on the tail had a relative temperature difference of roughly $-20 \%$.

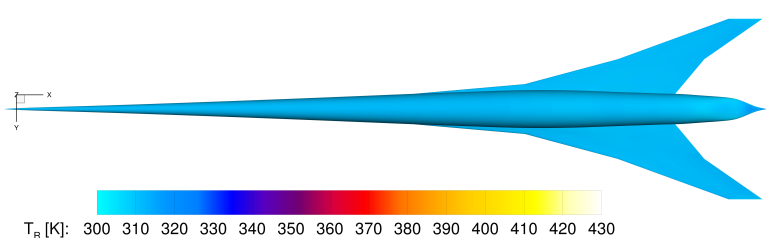

(a) Inviscid: Surface Recovery Temperatures

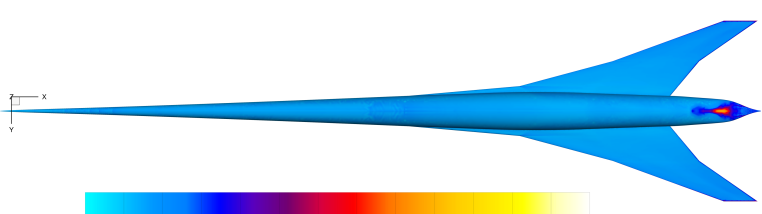

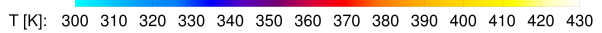

(b) Laminar: Surface Temperatures

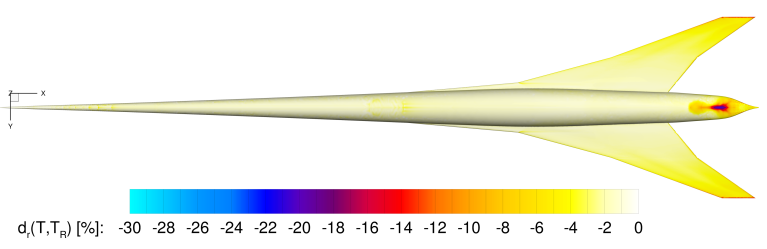

(c) Surface Temperature Percent Differences

Fig. 15 Comparing surface temperatures predicted using the medium mesh inviscid solution with laminar surface temperatures on the aircraft lower surface. 


\section{JAXA Geometry Perturbation Results}

The results from the previous sections indicate that the first $75-80 \%$ of the JAXA geometry can be accurately represented by the PANAIR solution. To determine if this section of the solution can be used in an optimization routine, a deformation was applied to the geometry. The deformation was defined as a Gaussian deformation with a center line located 7.74 meters from the nose and a maximum deformation of $3 \mathrm{~cm}$. This new geometry will be referred to as the JWB-bump geometry throughout the rest of this work. The coarse mesh used in the original JWB inviscid solution as well as the PANAIR surface mesh were both deformed to model the deformation. Figure 16 shows the altered surface meshes for PANAIR and UNS3D. Ten panels were added to the nose of the PANAIR mesh to accommodate the introduction of the deformation, which necessitated removing one section of azimuthal panels.

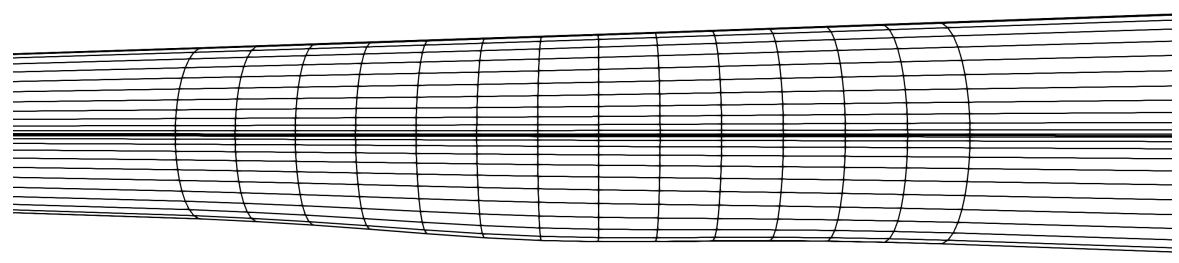

(a) Structured PANAIR mesh.

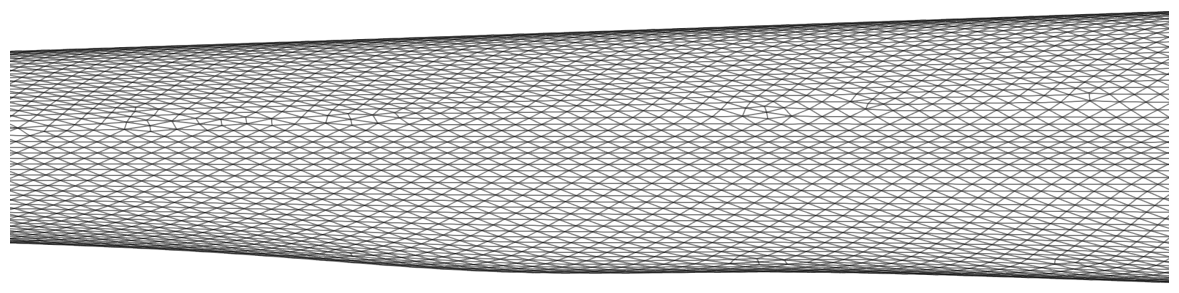

(b) Unstructured coarse UNS3D mesh.

Fig. 16 Altered computational meshes for the JWB-bump geometry.

The near-field pressure signature was extracted at $R / L=3$ and $\Phi=0^{\circ}$ for both the PANAIR solution and the inviscid solution. The results are shown in Fig. 17 for 32.92 meters of the signature. The rear part of the signature is characterized by the same pressure oscillations observed in the baseline case.

In comparison to the near-field pressure predicted by the UNS3D inviscid solution, PANAIR over-predicted the amplitudes of the new shocks generated by the deformation, as shown in Fig. 17 The over-prediction of the pressure could be the result of non-ideal paneling in the vicinity of the deformation. A grid convergence study in PANAIR in the region of the deformation could offer improvement to the solution in this region.

After propagating both signatures to the ground, many of the discrepancies between the the inviscid solution and PANAIR were smoothed out, as shown in Fig. 18. The PANAIR results still show a lack of nonlinear aging, however the predictions made at the ground in regards to the inclusion of the deformation match remarkably well.

The ground signatures were analyzed using both PyLdB and LCASB. The PyLdB (and LCASB) computed loudness values were 86.91 PLdB (86.92 PLdB) for the PANAIR ground signature and 81.53 PLdB (81.56 PLdB) for the coarse inviscid signature. Again, excellent agreement was seen between the PyLdB and LCASB predictions, with a maximum absolute percent difference of $0.04 \%$. The difference between the loudness of the inviscid and PANAIR ground signatures was similar to that observed with the baseline geometry.

The change in loudness that resulted from the inclusion of the surface deformation, $\Delta \mathrm{PLdB}=\mathrm{PLdB}_{\text {def }}-\mathrm{PLdB}_{\text {base }}$, was examined for both models. PANAIR predicted a loudness increase of $\triangle \mathrm{PLdB}=0.38 \mathrm{~dB}$, while the coarse inviscid solution predicted an increase of $\triangle \mathrm{PLdB}=1.6 \mathrm{~dB}$. These values corresponded to a relative increase in loudness of $0.44 \%$ and $2 \%$ for the PANAIR and inviscid predictions, respectively. As a consequence of the large difference in the relative PLdB increase predicted by the two models, optimization on the forward portion of the aircraft using 


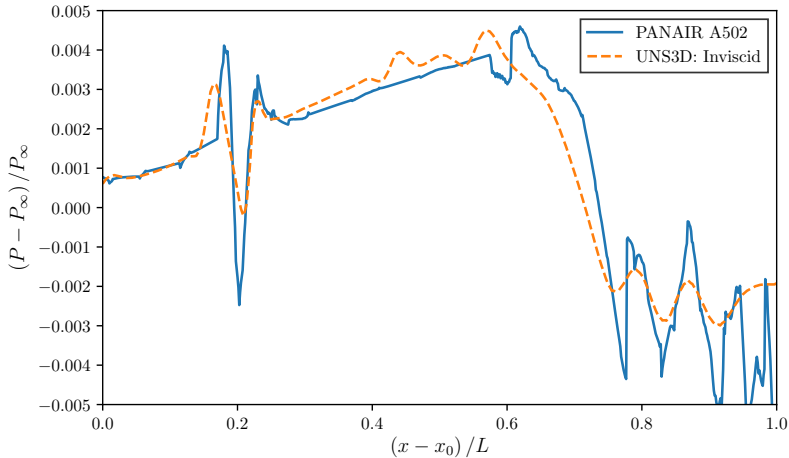

Fig. 17 Near-field pressure from PANAIR and coarse Euler solutions for the JWB-bump geometry.

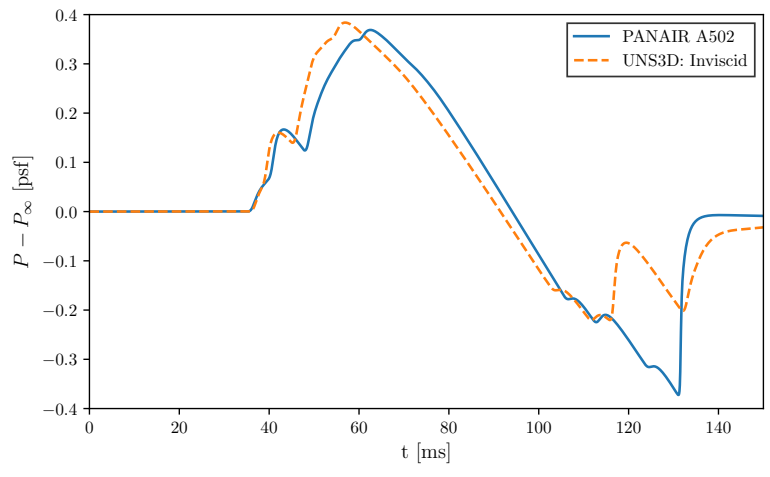

Fig. 18 Ground signatures from PANAIR and coarse Euler solutions for the JWB-bump geometry.

PANAIR,with the change in PLdB as the objective function, would likely result in a different optimum than if the higher-fidelity tool was used in the optimization process. However, further analysis must be performed to determine if the gradient of PLdB with respect to the location and size of the deformation are similar between the two models.

\section{Conclusions}

A panel convergence study was completed using PANAIR to determine the best number of panels and their placement on the geometry to achieve a grid independent solution. The surface pressures were found to be insensitive to span-wise refinement, except in the region directly adjacent to the wing tip. The wing was found to be sensitive to chord-wise refinement. The finest number of panels, clustered at the leading and trailing edges, was required to achieve the best surface solution. The tail portion of the aircraft was found to require a coarse axial spacing of the panels to prevent non-physical oscillations in pressure from appearing.

Examination of the velocity perturbation magnitudes in the PANAIR surface solution indicated that regions on the upper and lower surfaces were in violation of the Prandtl-Glauert equation's small velocity perturbation assumption. These regions of high perturbation velocity were the result of large surface curvature producing strong expansion shocks. Further analysis of these regions also indicated the violation of the "no transonic flow" assumption.

A comparison of the under-track pressure signatures three body lengths from the aircraft for each model was conducted. It was found that the PANAIR and UNS3D inviscid and laminar pressure signatures agreed well with each other along the first $0.8 \mathrm{~L}$ of the signature. Beyond this point, the PANAIR pressure signature was characterized by large oscillations in pressure that were not present in the high-fidelity predictions. The large pressure oscillations found in the PANAIR solution were caused by the aforementioned violations of the Prandtl-Glauert equations's fundamental assumptions. Flow separation predicted in the laminar solution caused a delayed, sharp re-compression of the near-field pressure. The UNS3D inviscid solution, however, did not predict flow separation.

Propagation of the under-track near-field signatures highlighted the lack of non-linear aging in the PANAIR solution. Despite the missing non-linear effects in the PANAIR solution, good qualitative agreement for the first half of the ground signature was found between the low- and high-fidelity methods. The predicted loudness of the UNS3D inviscid ground signatures agreed well with the published data. The large near-field pressure oscillations present in the PANAIR solution resulted in an increased peak underpressure, giving an increase of 6.6 PLdB over the UNS3D inviscid value. The sharp re-compression of the laminar near-field signature resulted in the flattening of the peak underpressure, leading to a 4.1 PLdB quieter sonic boom than the UNS3D inviscid boom. The difference in loudness due to the addition of viscous flow effects observed in this study was consistent with published data, which highlights the need for viscous flow features in the near-field solution to achieve realistic predictions for sonic boom loudness.

Both the PANAIR and UNS3D inviscid flow solvers were exercised to predict the sonic boom loudness of the low-boom geometry with an added surface deformation to asses their use for the optimization framework. The impact of the added surface deformation predicted by PANAIR to the near-field and ground signatures agreed qualitatively with the UNS3D inviscid prediction. However, the resultant variation in sonic boom loudness was not comparable between the two flow solvers. The relative increase in loudness predicted by PANAIR was $80 \%$ less than that predicted 
by UNS3D. In conclusion, the usefulness of the PANAIR solver in predicting the sonic boom loudness is limited by the assumptions of the Prandtl-Glauert equation.

\section{Acknowledgments}

The authors would like to thank the High Performance Research Computing center at Texas A\&M University for providing the computational resources that were required to complete the work presented in this paper. This work is supported by the NASA University Leadership Initiative (ULI) program under federal award number NNX17AJ96A, titled "Adaptive Aerostructures for Revolutionary Civil Supersonic Transportation".

\section{References}

[1] Friedlander, D. J., Heath, C., and Castner, R. S., "Numerical Simulations of a Quiet SuperSonic Technology (QueSST) Aircraft Preliminary Design," 2018 Fluid Dynamics Conference, American Institute of Aeronautics and Astronautics, 2018. doi:10.2514/6.2018-3861, URL https://doi.org/10.2514\%2F6.2018-3861.

[2] Risen, T., "Stakes Raised for NASA's Planned Supersonic X-Plane," Aerospace America, 2018. URL https:// aerospaceamerica.aiaa.org/departments/stakes-raised-for-nasas-planned-supersonic-x-plane/

[3] Magee, T. E., Fugal, S. R., Fink, L. E., Adamson, E. E., and Shaw, S. G., "System-Level Experimental Validations for Supersonic Commercial Transport Aircraft Entering Service in the 2018-2020 Time Period: Phase II Final Report," Technical Report NASA/CR-2015-218983, NASA Langley Research Center, 2015.

[4] Morgenstern, J., Buonanno, M., Yao, J., Murugappan, M., Paliath, U., Cheung, L., Ramakrishnan, I. M. K., Pastouchenko, N., and Wood, T., "Advanced Concept Studies for Supersonic Commercial Transports Entering Service in the 2018-2020 Period Phase 2,” Technical Report NASA/CR-2015-218719, NASA Langley Research Center, 2015.

[5] Maglieri, D. J., Bobbitt, P. J., Plotkin, K. J., Shepherd, K. P., Coen, P. G., and Richwine, D. M., "Sonic Boom: Six Decades of Research,” Technical Report NASA/SP-2014-622, NASA Langley Research Center, 2014.

[6] Chan, M. K., "Supersonic aircraft optimization for minimizing drag and sonic boom," PhD Dissertation, Stanford, California, 2003.

[7] Rallabhandi, S., and Mavris, D., "Design and analysis of supersonic business jet concepts," 6th AIAA Aviation Technology, Integration and Operations Conference (ATIO), 2006, p. 7702.

[8] Choi, S., Alonso, J. J., and Kroo, I. M., "Two-level multifidelity design optimization studies for supersonic jets," Journal of Aircraft, Vol. 46, No. 3, 2009, pp. 776-790.

[9] Galvan, E., and Malak, R. J., "P3GA: an algorithm for technology characterization," Journal of Mechanical Design, Vol. 137, No. 1, 2015, p. 011401.

[10] Hartl, D. J., Galvan, E., Malak, R. J., and Baur, J. W., "Parameterized design optimization of a magnetohydrodynamic liquid metal active cooling concept," Journal of Mechanical Design, Vol. 138, No. 3, 2016, p. 031402.

[11] Galvan, E., Malak, R. J., Hartl, D. J., and Baur, J. W., "Performance assessment of a multi-objective parametric optimization algorithm with application to a multi-physical engineering system," Structural and Multidisciplinary Optimization, 2018, pp. $1-21$.

[12] Giblette, T., and Hunsaker, D. F., "Prediction of Sonic Boom Loudness Using High-Order Panel Methods for the Near-Field Solution," AIAA Scitech 2019 Forum, 2019, p. 0605.

[13] Han, Z.-X., and Cizmas, P. G., "A CFD Method for Axial Thrust Load Prediction of Centrifugal Compressors," International Journal of Turbo \& Jet-Engines, Vol. 20, No. 1, 2003, pp. 1-16. doi:10.1515/tjj.2003.20.1.1, URL http://dx.doi.org/10. $1515 / \mathrm{tjj} .2003 .20 .1 .1$

[14] Carpenter, F. L., Cizmas, P. G., Reddy, S. R., and Dulikravich, G. S., "Controlling Sonic Boom Loudness Through Outer Mold Line Modification: A Sensitivity Study," AIAA Scitech 2019 Forum, American Institute of Aeronautics and Astronautics, 2019. doi:10.2514/6.2019-0603, URL https://doi.org/10.2514\%2F6.2019-0603.

[15] Leal, P. B., Giblette, T., Hunsaker, D. F., and Hartl, D. J., "Extended 3D Class/Shape Transformation equations for multicomponent aircraft assemblies," AIAA Scitech 2019 Forum, 2019, p. 0604. 
[16] Ehlers, F., Johnson, F., and Rubbert, P., "A higher order panel method for linearized supersonic flow," 9th Fluid and PlasmaDynamics Conference, 1979, p. 381.

[17] Epton, M. A., and Magnus, A. E., "PAN AIR: A computer program for predicting subsonic or supersonic linear potential flows about arbitrary configurations using a higher order panel method. Volume 1: Theory document (version 3.0)," 1990.

[18] Anderson, J. D., Modern Compressible Flow, $3^{\text {rd }}$ ed., McGraw Hill, 2003.

[19] Roe, P. L., “Approximate Riemann Solvers, Parameter Vectors, and Difference Schemes,” Journal of Computational Physics, Vol. 43, 1981, pp. 357-372. doi:10.1016/0021-9991(81)90128-5.

[20] Harten, A., and Hyman, J. M., "Self Adjusting Grid Methods for One-Dimensional Hyperbolic Conservation Laws," Journal of Computational Physics, Vol. 50, 1983, pp. 235-269. doi:10.1016/0021-9991(83)90066-9.

[21] Barth, T. J., and Jespersen, D. C., "The Design and Application of Upwind Schemes on Unstructured Meshes," 27th Aerospace Sciences Meeting, AIAA, 1989. doi:10.2514/6.1989-366.

[22] Carpenter, F. L., and Cizmas, P. G. A., "Transonic Fan Performance Evaluated With Different Solution Limiters," Volume 2B: Turbomachinery, ASME, 2017. doi:10.1115/gt2017-65174, URL https://doi .org/10.1115\%2Fgt2017-65174

[23] Haselbacher, A., and Blazek, J., "Accurate and Efficient Discretization of Navier-Stokes Equations on Mixed Grids," AIAA Journal, Vol. 38, No. 11, 2000, pp. 2094-2102. doi:10.2514/2.871.

[24] Saad, Y., and Schultz, M. H., "GMRES: A Generalized Minimal Residual Algorithm for Solving Nonsymmetric Linear Systems," SIAM Journal on Scientific and Statistical Computing, Vol. 7, No. 3, 1986, pp. 856-869. doi:10.1137/0907058, URL http://dx.doi.org/10.1137/0907058

[25] Blazek, J., Computational Fluid Dynamics: Principles and Applications, $2^{\text {nd }}$ ed., Elsevier, 2005.

[26] Rallabhandi, S. K., "Advanced Sonic Boom Prediction Using the Augmented Burgers Equation,” Journal of Aircraft, Vol. 48, No. 4, 2011, pp. 1245-1253. doi:10.2514/1.c031248, URL https://doi.org/10.2514\%2F1.c031248

[27] Pilon, A. R., "Spectrally accurate prediction of sonic boom signals," AIAA journal, Vol. 45, No. 9, 2007, pp. 2149-2156.

[28] Robinson, L. D., “Sonic Boom Propagation Through AN Inhomogeneous, Windy Atmosphere.” 1991.

[29] Cleveland, R. O., "Propagation of sonic booms through a real, stratified atmosphere," Ph.D. thesis, The University of Texas at Austin, 1995.

[30] U.S. Standard Atmosphere, 1976, U.S. Government Printing Office, 1976.

[31] Stevens, S., "Perceived level of noise by Mark VII and decibels (E)," The Journal of the Acoustical Society of America, Vol. 51, No. 2B, 1972, pp. 575-601.

[32] Leatherwood, J. D., Sullivan, B. M., Shepherd, K. P., McCurdy, D. A., and Brown, S. A., "Summary of recent NASA studies of human response to sonic booms," The Journal of the Acoustical Society of America, Vol. 111, No. 1, 2002, pp. 586-598.

[33] Loubeau, A., Naka, Y., Cook, B. G., Sparrow, V. W., and Morgenstern, J. M., "A new evaluation of noise metrics for sonic booms using existing data," AIP Conference Proceedings, Vol. 1685, AIP Publishing, 2015, p. 090015.

[34] Shepard, K. P., and Sullivan, B. M., "A Loudness Calculation Procedure Applied to Shaped Sonic Booms,” Technical Paper 3141, NASA, November 1991.

[35] Bolander, C. R., Hunsaker, D. F., Shen, H., and Carpenter, F. L., "Procedure for the Calculation of the Perceived Loudness of Sonic Booms," AIAA Scitech 2019 Forum, 2019, p. 2091.

[36] Ueno, A., Kanamori, M., and Makino, Y., "Multi-fidelity Low-boom Design Based on Near-field Pressure Signature,” 54th AIAA Aerospace Sciences Meeting, American Institute of Aeronautics and Astronautics, 2016. doi:10.2514/6.2016-2033.

[37] Park, M. A., and Nemec, M., "Near Field Summary and Statistical Analysis of the Second AIAA Sonic Boom Prediction Workshop," 35th AIAA Applied Aerodynamics Conference, American Institute of Aeronautics and Astronautics, 2017. doi:10.2514/6.2017-3256, URL https://doi.org/10.2514\%2F6.2017-3256.

[38] Saaris, G. R., Tinoco, E., Lee, J., and Rubbert, P., "A502I User's Manual-PAN AIR Technology Program for Solving Problems of Potential Flow about Arbitrary Configurations," Boeing Document, , No. D6-54703, 1992.

[39] Monaghan, R. J., "A Survey and Correlation of Data on Heat Transfer by Forced Convection at Supersonic Speeds," Ministry of Supply: Aeronautical Research Council Reports and Memoranda, , No. 3033, 1958. 